\title{
Atuação de profissionais de saúde e qualidade das ações no controle de câncer cervicouterino: um estudo transversal
}

\author{
Professionals' practice and quality of actions to control cervical cancer: a cross-sectional study \\ Tiempo de trabajo de profesionales y calidad de las acciones de control del cáncer cérvicouterino: un \\ estudio transversal
}

Eduarda Ferreira dos Anjos $^{1}$ (B) Kaue Batista Andrade ${ }^{2}$ (c)

Poliana Cardoso Martins ${ }^{1}$ (B) Jamille Amorim Carvalho Paiva ${ }^{3}$ (1) Nilia Maria de Brito Lima Prado ${ }^{1}$ (1)

Adriano Maia dos Santos ${ }^{1}$ (D)

1. Universidade Federal da Bahia, Instituto Multidisciplinar em Saúde, Mestrado em Saúde Coletiva. Vitória da Conquista, BA, Brasil.

2. Universidade Federal da Bahia, Instituto Multidisciplinar em Saúde. Vitória da Conquista, BA, Brasil.

3. Secretaria de Saúde do Estado da Bahia, Núcleo Regional de Saúde Sudoeste. Vitória da Conquista, BA, Brasil.

Autor correspondente:

Eduarda Ferreira dos Anjos.

E-mail: eduardaanjos93@gmail.com

Recebido em 01/05/2021.

Aprovado em 04/11/2021.

DOI:https://doi.org/10.1590/2177-9465-EAN-2021-0137

\section{Resumo}

Objetivo: avaliar o tempo de atuação de médicos e enfermeiros na Atenção Primária à Saúde (APS) e qualidade das ações desenvolvidas para controle do câncer cervicouterino (CC). Métodos: estudo transversal, conduzido de janeiro a março de 2019 em região de saúde compreendida em 19 municípios localizada no estado da Bahia, Brasil. A amostra foi de 241 médicos e enfermeiros da APS. Utilizou-se a linha de cuidado do CC como condição traçadora. Elegeram-se o desfecho tempo de atuação na APS no mesmo município, categorizado em $<2$ anos e $\geq 2$ anos, e indicadores representativos da qualidade da APS. Os testes $\chi^{2}$ de Pearson e exato de Fisher foram empregados. Resultados: a prevalência de tempo de atuação na APS foi 43,57\% (IC95\%: 37,40\%; 49,94\%) para < 2 anos e 56,43\% (IC95\%: 50,06\%; 62,60\%) para $\geq 2$ anos. Observaram-se maiores prevalências, com diferença estatística significativa, dos indicadores de qualidade para o maior tempo de atuação. Conclusões e implicações para a prática: a rotatividade profissional parece afetar o cuidado longitudinal de mulheres na linha de cuidado eleita. Sugere-se a ampliação do número e do papel dos enfermeiros, especialmente nos serviços de APS, para maior resolutividade e eficiência do sistema de saúde.

Palavras-chave: Atenção Primária à Saúde; Mão de Obra em Saúde; Neoplasias do Colo do Útero; Qualidade da Assistência à Saúde; Regionalização.

\section{Abstract}

Objective: to assess the working length of physicians and nurses in Primary Health Care (PHC) and the quality of actions taken to control cervical cancer (CC). Methods: this is a cross-sectional study, conducted from January to March 2019, in a health region comprised of 19 municipalities located in the state of Bahia, Brazil. The sample consisted of $241 \mathrm{PHC}$ physicians and nurses. The CC care line was used as a tracer condition. The outcome experience length in PHC in the same municipality was chosen, categorized as $<2$ years and $\geq 2$ years and representative indicators of PHC quality. Pearson's $\chi 2$ and Fisher's exact tests were used. Results: the prevalence of length of experience in $\mathrm{PHC}$ was $43.57 \%$ (95\% $\mathrm{Cl}: 37.40 \% ; 49.94 \%)$ for < 2 years, and $56.43 \%$ (95\% $\mathrm{Cl}: 50.06 \% ; 62.60 \%$ ) for $\geq 2$ years. There was a higher prevalence, with a statistically significant difference, of the quality indicators for the longest working length. Conclusions and implications for practice: professional turnover seems to affect the longitudinal care of women in the chosen care line. It is suggested to expand the number and role of nurses, especially in $\mathrm{PHC}$ services, for greater resolution and efficiency of the health system.

Keywords: Primary Health Care; Health Workforce; Cervical Cancer; Quality of Health Care; Regional Health Planning.

\section{Resumen}

Objetivo: evaluar el tiempo de actuación de médicos y enfermeros en la Atención Primaria de Salud (APS) y la calidad de las acciones desarrolladas para el control del cáncer cérvicouterino (CC). Métodos: estudio transversal realizado de enero a marzo de 2019, en una región sanitaria que comprende 19 municipios en el estado de Bahía, Brasil. La muestra fue de 241 médicos y enfermeros de APS. La línea de cuidados de CC fue la condición trazadora. Se eligió el resultado tiempo trabajando en APS en el mismo municipio, categorizado en $<2$ años $y \geq 2$ años e indicadores representativos de calidad de APS. Se utilizaron pruebas exactas de chi-cuadrado de Pearson y Fisher. Resultados: la prevalencia del tiempo de actuación en APS fue del 43,57\% (IC95\%: 37,40\%; 49,94\%) para < 2 años y del 56,43\% (IC95\%: 50,06\%; 62,60\%) para $\geq 2$ años, considerado incipiente. Se observó una mayor prevalencia, con diferencia estadísticamente significativa, de los indicadores de calidad para un mayor tiempo de actuación. Conclusiones e implicaciones para la práctica: la rotación de profesionales parece afectar la atención longitudinal de las mujeres en la línea de cuidado elegida. Se sugiere la ampliación del número y el papel de enfermería, especialmente en los servicios de APS, para una mayor resolutividad y eficiencia del sistema sanitario.

Palabras clave: Atención Primaria de Salud; Fuerza Laboral en Salud; Neoplasias del Cuello Uterino; Calidad de la Atención de Salud; Regionalización. 


\section{INTRODUÇÃO}

O câncer cervicouterino (CC) é um grave problema de saúde pública, pois ainda se constitui uma das principais causas de morte entre as mulheres em todo o mundo ${ }^{1,2}$. Acomete, principalmente, adultas jovens, que têm muitas responsabilidades econômicas e de cuidados com suas famílias ${ }^{2}$. A doença tem distribuição heterogênea, sendo mais incidente em países de renda baixa e média, evidenciando diferentes exposições aos fatores de risco, contexto econômico, estilo de vida e acesso a cuidados aos serviços de saúde'.

O CC é um importante marcador de iniquidade em saúde $e^{3,4}$, pois é uma doença de curso longo, altamente evitável e um problema controlado em países com vacinação, rastreamento e tratamento consolidados ${ }^{5}$. Nesse sentido, a Atenção Primária à Saúde (APS), na lógica regional, constitui-se estratégia principal para lidar com doenças crônicas ${ }^{6}$.

No Brasil, assim como no panorama global, a distribuição do CC se dá de maneira dispare entre as regiões, havendo maiores prevalências no Norte e Nordeste ${ }^{5}$. A região de saúde eleita para o estudo está localizada no estado da Bahia, no Nordeste, e reflete, portanto, tal cenário. A região é composta majoritariamente por municípios com pouca capacidade econômica, baixa taxa de urbanização, grande extensão territorial e estagnação ou diminuição do contingente populacional, que significam importantes barreiras sinérgicas para atração e fixação de profissionais ${ }^{7}$.

Em municípios rurais e de difícil acesso, há grande quantidade de mulheres vulneráveis e altas taxas de neoplasias do colo do útero ${ }^{2-4,8-10}$. Em contrapartida, encontram dificuldade de atração e fixação de profissionais ${ }^{6}$, especialmente médicos ${ }^{11}$. Essa alta rotatividade dificulta a longitudinalidade do cuidado e a formação de vínculo ${ }^{12,13}$, gerando disparidades persistentes no acesso aos serviços ${ }^{8}$ e prejuízos na qualidade da assistência à saúde ${ }^{14}$.

A disponibilidade e a distribuição de profissionais se mostram desigual em todo o mundo, e, apesar de não haver definição de período mínimo adequado para atuação na APS, o fortalecimento da força de trabalho é fundamental para transformação dos modelos de atenção à saúde ${ }^{15}$. É, portanto, basilar para influenciar na determinação social, equipes multiprofissionais vinculadas à comunidade e que conheçam seus territórios de atuação ${ }^{7}$.

No âmbito da APS, os enfermeiros são mais envolvidos na busca e no rastreamento de mulhere ${ }^{16}$, favorecendo a construção de uma relação de confiança ${ }^{17,18}$. Nessa perspectiva, torna-se um sujeito fundamental para manutenção do acompanhamento ${ }^{19}$ e percepção positiva dos usuários em relação à assistência ${ }^{20}$.

A expansão das funções do enfermeiro na $\operatorname{APS}^{21}$ e a capacitação dos profissionais ${ }^{14,20}$ contribuem com a qualidade dos serviços, refletindo em melhor integralidade ${ }^{22}$ e resolutividade da rede de atenção à saúde. Assim, auxiliam para minimização de barreiras de acesso geográficas, organizacionais, estruturais e simbólicas, que podem influenciar negativamente na triagem de mulheres ${ }^{17,18}$.

A definição de qualidade de serviços se torna complexa, ao se ponderar a polissemia do termo. Para adotar um conceito claro e conciso de qualidade da APS, a Política Nacional de
Atenção Básica e o Programa Nacional de Melhoria do Acesso e da Qualidade da Atenção Básica (PMAQ-AB) trazem aspectos essenciais para adequada prestação do cuidado da $\mathrm{APS}^{7,23}$. A partir de tais pressupostos, elegeram-se indicadores para avaliação da qualidade dos serviços prestados na APS na linha de cuidado de saúde da mulher.

Além de notadamente influenciar na qualidade da APS, a melhor retenção de profissionais gera menos custos ao sistema de saúde e melhores resultados para a população ${ }^{10}$. Nesse sentido, o presente estudo buscou avaliar o tempo de atuação de médicos e enfermeiros na APS e a qualidade dos serviços prestados, utilizando o CC como condição traçadora.

\section{MÉTODOS}

Estudo transversal, realizado de janeiro a março de 2019. Elegeu-se a linha de cuidado do CC para avaliar a qualidade dos serviços prestados ${ }^{24}$ na APS da região de saúde de Vitória da Conquista, Bahia, Brasil. Essa região de saúde é composta por 19 municípios com, aproximadamente, 33\% da população residindo na zona rural. No momento da coleta, existiam 177 Equipes de Saúde da Família (EqSF), sendo 83 de zona urbana e 94 de zona rural, compostas por 354 médicos e enfermeiros, atuando em territórios de vasta extensão territorial e muitas mulheres em vulnerabilidade social (Tabela 1).

A amostra foi calculada com a adoção da prevalência de $50 \%$ para eventos desconhecidos, poder de $80 \%$ e nível de confiança de $95 \%$, obtendo-se o número mínimo de 240 médicos e enfermeiros, considerando $30 \%$ para eventuais perdas. Após o cálculo amostral, a seleção dos entrevistados foi realizada com base em sorteio aleatório, levando em conta o número proporcional de médicos e enfermeiros cadastrados nas EqSF de cada município. O critério de inclusão foi ser médico ou enfermeiro das EqSF dos 19 municípios da região de saúde. Os profissionais que não estavam atuando no período da coleta (férias, licenças, etc.) foram excluídos.

Elegeram-se médicos e enfermeiros das EqSF, por serem os responsáveis pela coleta do exame citopatológico nas Unidades de Saúde da Família (USF), além de realizarem o acompanhamento das pacientes em casos de lesão precursoras e CC. Sob essa perspectiva, médicos e enfermeiros operam a gestão da linha de cuidado na micropolítica do trabalho em saúde, por meio de ações de promoção da saúde e monitoramento do controle do CC.

Foram realizadas entrevistas por meio de aplicação de questionários por entrevistadores devidamente treinados, com utilização de tablets. O questionário elaborado foi adaptado de instrumento aplicado em pesquisa na $\mathrm{Bahia}^{25}$, no qual formam agregadas informações de protocolos do Instituto Nacional do Câncer (INCA) ${ }^{5}$ e dos Cadernos de Atenção Básica ${ }^{23}$. Utilizou-se o software KoboTollbox ${ }^{\circledR}$, versão 1.4 .8 , para a programação e armazenamento dos dados. Realizou-se estudo piloto em município de região de saúde vizinha, para adequação do instrumento de pesquisa, da logística e organização do campo. Tais dados não foram utilizados para análise. 
Tabela 1. Características sociodemográficas e de saúde, região de saúde de Vitória da Conquista, Bahia, Brasil, 2019.

\begin{tabular}{|c|c|c|c|c|c|c|}
\hline Localidade & $\begin{array}{l}\text { Distância } \\
\text { à Sede }\end{array}$ & $\begin{array}{l}\text { Pop. ZR/ZU } \\
(2010)^{*}\end{array}$ & $\begin{array}{l}\text { IDHM } \\
(2010)^{*}\end{array}$ & $\begin{array}{l}\text { Pop. Beneficiada } \\
\text { pelo PBF }(2019)^{+}\end{array}$ & $\begin{array}{l}\text { Cobertura de } \\
\text { ESF }(2018)^{\ddagger}\end{array}$ & $\begin{array}{l}\text { Número de } \\
\text { EqSF }(2018)^{\S}\end{array}$ \\
\hline Maetinga & $130 \mathrm{~km}$ & $4.221 / 2.817$ & 0,538 & $39,2 \%$ & $100 \%$ & ZR:04/ZU:01 \\
\hline Ribeirão do Largo & $109 \mathrm{~km}$ & $3.955 / 4.647$ & 0,540 & $45,1 \%$ & $100 \%$ & ZR:03/ZU:01 \\
\hline Cordeiros & $162 \mathrm{~km}$ & $5.617 / 2.551$ & 0,579 & $28,8 \%$ & $100 \%$ & ZR:02/ZU:02 \\
\hline Mirante & $139 \mathrm{~km}$ & $8.698 / 1.809$ & 0,527 & $31,6 \%$ & $100 \%$ & ZR:04/ZU:01 \\
\hline Caraíbas & $82,7 \mathrm{~km}$ & $7.709 / 2.513$ & 0,555 & $35,9 \%$ & $100 \%$ & ZR:03/ZU:01 \\
\hline Bom Jesus da Serra & $95,1 \mathrm{~km}$ & $7.345 / 2.768$ & 0,546 & $39,4 \%$ & $100 \%$ & ZR:02/ZU:02 \\
\hline Piripá & $124 \mathrm{~km}$ & $6.588 / 6.195$ & 0,575 & $28,5 \%$ & $100 \%$ & ZR:04/ZU:02 \\
\hline Presidente Jânio Quadros & $122 \mathrm{~km}$ & $9.454 / 4.198$ & 0,542 & $37,3 \%$ & $100 \%$ & ZR:04/ZU:03 \\
\hline Caetanos & $72,5 \mathrm{~km}$ & $10.348 / 3.291$ & 0,542 & $43,1 \%$ & $100 \%$ & ZR:05/ZU:01 \\
\hline Tremedal & $82,5 \mathrm{~km}$ & $13.090 / 3.939$ & 0,528 & $33,9 \%$ & $100 \%$ & ZR:04/ZU:03 \\
\hline Belo Campo & $63,7 \mathrm{~km}$ & $6.992 / 9.029$ & 0,575 & $38,7 \%$ & $100 \%$ & ZR:03/ZU:03 \\
\hline Condeúba & $149 \mathrm{~km}$ & $9.436 / 7.462$ & 0,582 & $32,8 \%$ & $100 \%$ & ZR:05/ZU:03 \\
\hline Encruzilhada & $97,9 \mathrm{~km}$ & $18.636 / 5.130$ & 0,544 & $33,2 \%$ & $100 \%$ & ZR:06/ZU:02 \\
\hline Anagé & $52,3 \mathrm{~km}$ & $20.592 / 4.924$ & 0,540 & $36,3 \%$ & $100 \%$ & ZR:08/ZU:03 \\
\hline Cândido Sales & $85,5 \mathrm{~km}$ & $8.632 / 19.286$ & 0,601 & $33,8 \%$ & $100 \%$ & ZR:03/ZU:07 \\
\hline Planalto & $47,5 \mathrm{~km}$ & $9.612 / 14.869$ & 0,560 & $33,9 \%$ & $100 \%$ & ZR:06/ZU:03 \\
\hline Barra do Choça & $30,7 \mathrm{~km}$ & $12.381 / 22.407$ & 0,551 & $39,4 \%$ & $100 \%$ & ZR:06/ZU:06 \\
\hline Poções & $69,1 \mathrm{~km}$ & $10.042 / 34.659$ & 0,604 & $37,7 \%$ & $100 \%$ & ZR:04/ZU:11 \\
\hline Vitória da Conquista & Sede & $32.127 / 274.739$ & 0,678 & $16,9 \%$ & $44,5 \%$ & ZR:18/ZU:28 \\
\hline Região de saúde & - & $205.475 / 422.309$ & - & - & $71,1 \%$ & ZR:94/ZU:83 \\
\hline
\end{tabular}

O tempo de atuação na APS no mesmo município foi o desfecho considerado no estudo, dicotomizado em $<2$ anos $\mathrm{e} \geq 2$ anos. Para avaliar a qualidade na APS, foram utilizadas as variáveis independentes: função (médico; enfermeiro); sexo (masculino; feminino); idade (20-29 anos; 30-39 anos; $\geq 40$ anos); pós-graduação (sim; não); EqSF completas (sim; não); frequência de oferta do exame citopatológico (mensal; quinzenal; semanal ou mais); realização de mutirões que, no contexto da APS, são ações coletivas para ampliação do acesso aos serviços oferecidos nas chamadas semanas típicas, diminuindo filas de espera para realização do exame preventivo (sim; não); monitoramento do registro para identificar mulheres com coleta atrasada (sim; não); seguimento das mulheres durante tratamento na atenção especializada (sim; não); acesso ao transporte sanitário fornecido pela Secretaria Municipal de Saúde (nunca/às vezes; sempre); existência de mulheres na unidade diagnosticadas com CC no últimos 12 meses (sim; não); e diagnóstico de lesão de alto grau (HSIL) (sim; não) (Figura 1).

A escolha das variáveis explicativas, consideradas indicadores de qualidade da APS, teve como base o instrumento de avaliação externa do PMAQ-AB. Nesse sentido, além de possibilitar a análise de questões do resultado e do processo de trabalho da APS, permite a avaliação de aspectos da estrutura, para oportunizar a concretização dos atributos de longitudinalidade, coordenação do cuidado, integralidade, utilizando a linha de cuidado da saúde da mulher ${ }^{14}$.

As análises descritivas foram obtidas por meio das medidas de frequência absolutas ( $n$ ) e relativas (\%). As diferenças entre as proporções foram avaliadas pelos testes $\chi^{2}$ de Pearson e exato de Fisher. Realizaram-se análises ajustadas para sexo, idade e estado conjugal, tendo em consideração que tais variáveis podem atuar como potenciais confundidoras para a fixação e o tempo de atuação do profissional. Considerou-se um $\mathrm{p}$-valor $\leq 0,05$ e utilizou-se o pacote estatístico Stata (Stata Corporation, College Station, USA), versão 15.0, para análise dos dados.

Foi realizada análise descritiva das variáveis, por nível assistencial, por meio de frequências

A pesquisa foi aprovada pelo Comitê de Ética em Pesquisa da Universidade Federal da Bahia, sob Parecer no 624.168, de 24 de abril de 2014 e CAAE no 27247414.0.0000.5556. Todos os 


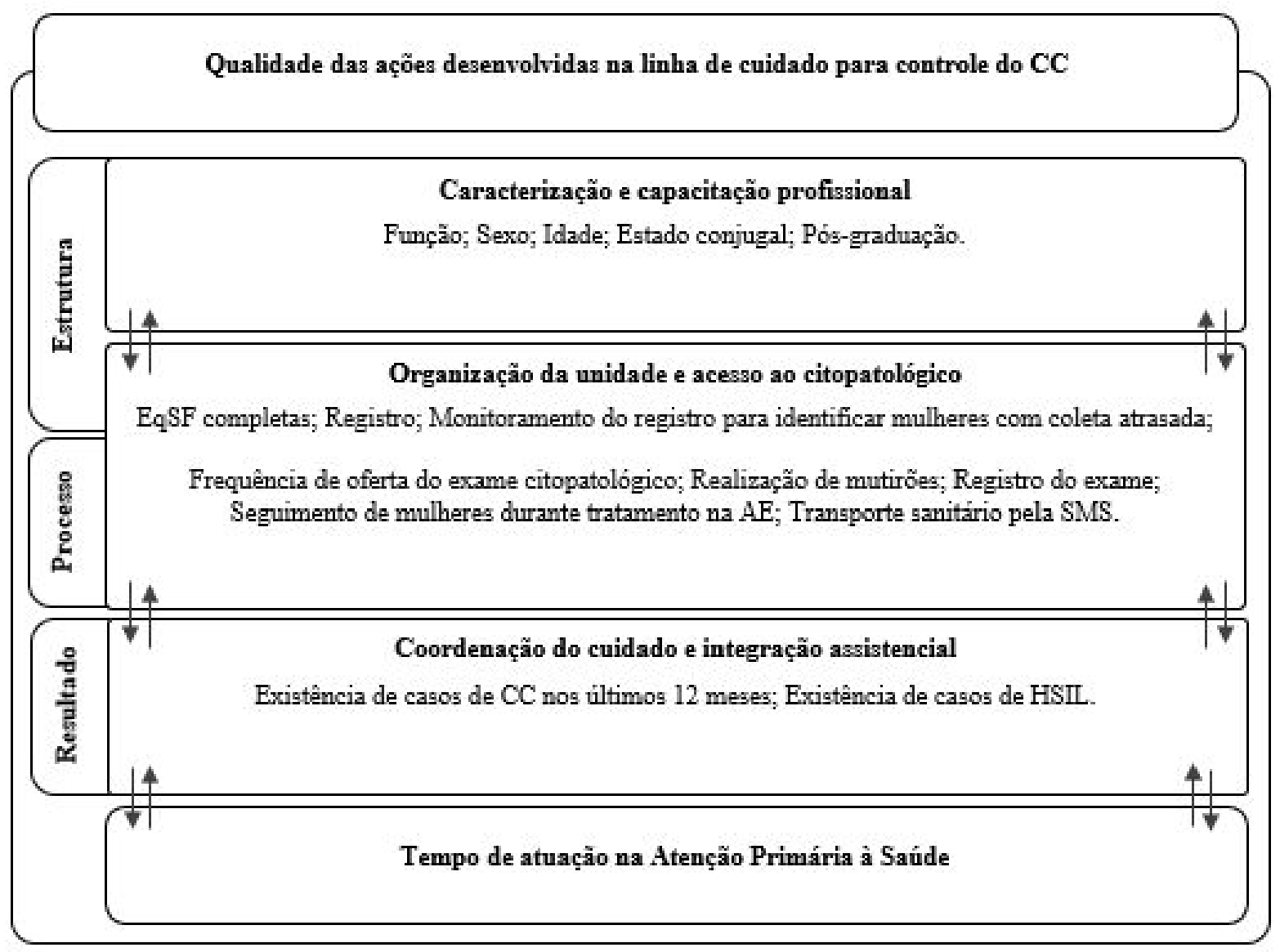

Figura 1. Modelo conceitual de análise do tempo de atuação e qualidade das ações desenvolvidas na linha de cuidado para controle do câncer cervicouterino.

CC - câncer cervicouterino; EqSF - Equipes de Saúde da Família; AE - atenção especializada; SMS - Secretaria Municipal de Saúde; HSIL - lesão intraepitelial de alto grau. Fonte: elaboração própria com base no referencial teórico realizado para pesquisa.

participantes forneceram consentimento escrito à participação na pesquisa. Foram seguidos os preceitos éticos da Resolução no 466/2012 do Conselho Nacional de Saúde.

\section{RESULTADOS}

A prevalência do tempo de atuação na APS no mesmo município foi de 43,57\% (IC95\%: 37,40;49,94) para <2 anos e de $56,43 \%$ (IC95\%: 50,06;62,60) para $\geq 2$ anos. Foram entrevistados 241 profissionais (109 médicos e 132 enfermeiros), sendo a maioria do sexo feminino $(71,8 \%)$, com idade entre 30 e 39 anos $(47,7 \%)$, sem companheiro(a) $(51,9 \%)$ e que haviam realizado pós-graduação $(67,2 \%)$ (Tabela 2$)$.

Com relação à organização da APS, grande parte dos profissionais relatou estar em equipes completas $(94,6 \%)$. A oferta de coleta do exame citopatológico do colo uterino era pelo menos uma vez por semana $(67,7 \%)$. Ainda assim, a maioria $(74,7 \%)$ relatou que as unidades eventualmente realizavam mutirões para ampliar o acesso ao Papanicolau. O registro era realizado em quase a totalidade das unidades $(96,7 \%)$ e fazia-se o monitoramento deste para identificar mulheres que pudessem estar com coletas atrasadas $(74,3 \%$ ) (Tabela 2 ).

Foi relatado, ainda, que as mulheres continuavam sendo acompanhadas por suas respectivas EqSF (76,7\%), mesmo durante o tratamento na atenção especializada e que as usuárias conseguiam acessar o transporte sanitário $(65,5 \%)$, ofertado pela Secretaria Municipal de Saúde. Entretanto, mais da metade dos profissionais indicou haver casos de mulheres com diagnóstico de HSIL $(53,6 \%$ ) em suas unidades de saúde (Tabela 2).

A comparação para os diferentes tempos de atuação e os indicadores de qualidade eleitos podem ser visualizados na Tabela 3. A análise mostrou que houve uma diferença entre a função exercida e o tempo de atuação, sendo mais comum o maior tempo entre os enfermeiros (68,2\%), comparando-se com os médicos $(42,2 \% ; p<0,001)$. Dentre os profissionais com o tempo de atuação $\geq 2$ anos, observam-se maiores prevalências, com diferença estatística para o sexo feminino $(69,5 \% ; p=0,004)$, idade $\geq 40$ anos $(76,8 \% ; p<0,001)$ e estado conjugal com 
Tabela 2. Caracterização das equipes e unidades de saúde da família, região de saúde de Vitória da Conquista, Bahia, 2019.

\begin{tabular}{|c|c|c|c|}
\hline \multirow{2}{*}{ Variável } & \multicolumn{3}{|c|}{ Distribuição da amostra } \\
\hline & $\mathbf{n}$ & $\%$ & $\mathrm{IC}_{95 \%}$ \\
\hline \multicolumn{4}{|c|}{ Caracterização e capacitação profissional } \\
\hline \multicolumn{4}{|l|}{ Função } \\
\hline Médico & 109 & 45,2 & $39,01-51,60$ \\
\hline Enfermeiro & 132 & 54,8 & $48,40-60,99$ \\
\hline \multicolumn{4}{|l|}{ Sexo } \\
\hline Masculino & 68 & 28,2 & $22,86-34,27$ \\
\hline Feminino & 173 & 71,8 & $65,73-77,14$ \\
\hline \multicolumn{4}{|l|}{ Idade } \\
\hline 20-29 anos & 70 & 29,1 & $23,63-35,14$ \\
\hline 30-39 anos & 115 & 47,7 & $41,44-54,07$ \\
\hline$\geq 40$ anos & 56 & 23,2 & $18,30-29,03$ \\
\hline \multicolumn{4}{|l|}{ Estado conjugal } \\
\hline Sem companheiro(a) & 125 & 51,9 & $45,52-58,16$ \\
\hline Com companheiro(a) & 116 & 48,1 & $41,84-54,48$ \\
\hline \multicolumn{4}{|l|}{ Pós-graduação } \\
\hline Não & 79 & 32,8 & $27,11-39,00$ \\
\hline Sim & 162 & 67,2 & $61,00-72,89$ \\
\hline \multicolumn{4}{|c|}{ Organização da USF e acesso ao citopatológico } \\
\hline \multicolumn{4}{|c|}{ EqSF completas } \\
\hline Não & 13 & 5,4 & $3,14-9,10$ \\
\hline Sim & 228 & 94,6 & $90,90-96,85$ \\
\hline \multicolumn{4}{|c|}{ Frequência de oferta do exame citopatológico } \\
\hline Mensal & 22 & 9,9 & $6,56-14,57$ \\
\hline Quinzenal & 50 & 22,4 & $17,39-28,41$ \\
\hline Semanal ou mais & 151 & 67,7 & $61,25-73,56$ \\
\hline \multicolumn{4}{|l|}{ Realização de mutirões } \\
\hline Não & 58 & 25,3 & $20,08-31,41$ \\
\hline Sim & 171 & 74,7 & $68,59-79,91$ \\
\hline \multicolumn{4}{|c|}{ Registro do exame citopatológico } \\
\hline Não & 8 & 3,3 & $1,66-6,53$ \\
\hline Sim & 233 & 96,7 & $93,47-98,34$ \\
\hline \multicolumn{4}{|c|}{$\begin{array}{l}\text { Monitoramento do registro para identificar } \\
\text { mulheres com coleta atrasada }\end{array}$} \\
\hline Não & 62 & 25,7 & $20,56-31,66$ \\
\hline Sim & 179 & 74,3 & $68,34-79,43$ \\
\hline \multicolumn{4}{|c|}{$\begin{array}{l}\text { Seguimento das mulheres durante tratamento } \\
\text { na AE }\end{array}$} \\
\hline Nunca ou às vezes & 52 & 23,3 & $18,19-29,37$ \\
\hline
\end{tabular}

IC95\% - intervalo de confiança de 95\%; USF - Unidade de Saúde da Família; EqSF - Equipe de Saúde da Família; AE - atenção especializada; SMS - Secretaria Municipal de Saúde; CC - câncer cervicouterino; HSIL - lesão intraepitelial de alto grau. Fonte: elaboração própria com base nos dados da pesquisa. 
Tabela 2. Continuação...

\begin{tabular}{|c|c|c|c|}
\hline \multirow{2}{*}{ Variável } & \multicolumn{3}{|c|}{ Distribuição da amostra } \\
\hline & $\mathbf{n}$ & $\%$ & $\mathrm{IC}_{95 \%}$ \\
\hline Sempre & 171 & 76,7 & $70,63-81,80$ \\
\hline \multicolumn{4}{|l|}{ Transporte sanitário pela SMS } \\
\hline Nunca ou às vezes & 69 & 34,5 & $28,19-41,41$ \\
\hline Sempre & 131 & 65,5 & $58,59-71,81$ \\
\hline \multicolumn{4}{|c|}{$\begin{array}{l}\text { Coordenação do cuidado e integração } \\
\text { assistencial }\end{array}$} \\
\hline \multicolumn{4}{|c|}{ Existência de casos de CC nos últimos 12 meses } \\
\hline Não & 150 & 68,8 & $62,30-74,65$ \\
\hline Sim & 68 & 31,2 & $25,35-37,70$ \\
\hline \multicolumn{4}{|l|}{ Existência de casos de HSIL } \\
\hline Não & 103 & 46,4 & $39,89-53,03$ \\
\hline Sim & 119 & 53,6 & $46,97-60,11$ \\
\hline
\end{tabular}

IC95\% - intervalo de confiança de 95\%; USF - Unidade de Saúde da Família; EqSF - Equipe de Saúde da Família; AE - atenção especializada; SMS - Secretaria Municipal de Saúde; CC - câncer cervicouterino; HSIL - lesão intraepitelial de alto grau. Fonte: elaboração própria com base nos dados da pesquisa.

Tabela 3. Tempo de atuação na atenção primária à saúde e indicadores de qualidade segundo dimensões, região de saúde de Vitória da Conquista, Bahia, 2019.

\begin{tabular}{|c|c|c|c|c|}
\hline Dimensões/variáveis & $<2$ anos $(105) n(\%)$ & $\geq 2$ anos $(136) n(\%)$ & Total (241) n (\%) & p-valor \\
\hline \multicolumn{5}{|c|}{ Caracterização e capacitação profissional } \\
\hline \multicolumn{5}{|l|}{ Função } \\
\hline Médico & $63(57,8)$ & $46(42,2)$ & $109(45,2)$ & \\
\hline Enfermeiro & $42(31,8)$ & $90(68,2)$ & $132(54,8)$ & $<0,001^{*}$ \\
\hline Sexo ${ }^{\ddagger}$ & $n=74$ & $n=123$ & $n=197$ & \\
\hline Masculino & $34(51,5)$ & $32(48,5)$ & $66(33,5)$ & \\
\hline Feminino & $40(30,5)$ & $91(69,5)$ & $131(66,5)$ & $0,004 *$ \\
\hline \multicolumn{5}{|l|}{ Idade } \\
\hline 20-29 anos & $47(67,1)$ & $23(32,9)$ & $70(29,0)$ & \\
\hline 30-39 anos & $45(39,1)$ & $70(60,9)$ & $115(47,7)$ & \\
\hline$\geq 40$ anos & $13(23,2)$ & $43(76,8)$ & $56(23,3)$ & $<0,001^{*}$ \\
\hline \multicolumn{5}{|l|}{ Estado conjugal } \\
\hline Sem companheiro(a) & $70(56,0)$ & $55(44,0)$ & $125(51,9)$ & \\
\hline Com companheiro(a) & $35(30,2)$ & $81(69,8)$ & $116(48,1)$ & $<0,001^{*}$ \\
\hline \multicolumn{5}{|l|}{ Pós-graduação } \\
\hline Não & $55(69,6)$ & $24(30,4)$ & $79(32,8)$ & \\
\hline Sim & $50(30,4)$ & $112(69,1)$ & $162(67,2)$ & $<0,001^{*}$ \\
\hline $\begin{array}{l}\text { Organização da USF e } \\
\text { citopatológico }\end{array}$ & & & & \\
\hline
\end{tabular}

*Teste Qui-quadrado de Pearson; 'Teste exato de Fisher; ${ }^{\ddagger}$ A variável sexo só mantém diferença estatística significativa após ajuste para idade e estado conjugal e a variável frequência de oferta do exame citopatológico só mantém diferença estatística significativa após ajuste para sexo, idade e estado conjugal; USF Unidade de Saúde da Família; EqSF - Equipe de Saúde da Família; AE - atenção especializada; SMS - Secretaria Municipal de Saúde; CC - câncer cervicouterino; HSIL - lesão intraepitelial de alto grau. Fonte: elaboração própria com base nos dados da pesquisa. 
Tabela 3. Continuação...

\begin{tabular}{|c|c|c|c|c|}
\hline \multicolumn{5}{|l|}{ EqSF completas } \\
\hline Não & $5(38,5)$ & $8(61,5)$ & $13(5,4)$ & \\
\hline Sim & $100(43,9)$ & $128(56,1)$ & $228(94,6)$ & $0,780^{+}$ \\
\hline $\begin{array}{l}\text { Frequência de oferta do exame } \\
\text { citopatológico }\end{array}$ & $n=82$ & $n=128$ & $n=210$ & \\
\hline Mensal & $14(63,6)$ & $8(36,4)$ & $22(10,5)$ & \\
\hline Quinzenal & $14(30,4)$ & $32(69,6)$ & $46(21,9)$ & \\
\hline Semanal ou mais & $54(38,0)$ & $88(62,0)$ & $142(67,6)$ & $0,033^{+}$ \\
\hline Realização de mutirões & $n=95$ & $n=134$ & $n=229$ & \\
\hline Não & $31(53,5)$ & $27(46,5)$ & $58(25,3)$ & \\
\hline Sim & $64(37,4)$ & $107(62,6)$ & $171(74,7)$ & $0,032 *$ \\
\hline \multicolumn{5}{|l|}{ Registro do exame citopatológico } \\
\hline Não & $7(87,5)$ & $1(12,5)$ & $8(3,3)$ & \\
\hline Sim & $98(42,1)$ & $135(57,9)$ & $233(96,7)$ & $0,023^{+}$ \\
\hline \multicolumn{5}{|l|}{$\begin{array}{l}\text { Monitoramento do registro para } \\
\text { identificar mulheres com coleta atrasada }\end{array}$} \\
\hline Não & $40(64,5)$ & $22(35,5)$ & $62(25,7)$ & \\
\hline Sim & $65(36,3)$ & $114(63,7)$ & $179(74,3)$ & $<0,001^{*}$ \\
\hline $\begin{array}{l}\text { Seguimento das mulheres durante } \\
\text { tratamento na } \mathrm{AE}\end{array}$ & $n=89$ & $n=134$ & $n=223$ & \\
\hline Nunca ou às vezes & $25(48,1)$ & $27(51,9)$ & $52(23,3)$ & \\
\hline Sempre & $64(37,4)$ & $107(62,6)$ & $171(76,7)$ & $0,170 *$ \\
\hline Transporte sanitário pela SMS & $n=85$ & $n=115$ & $n=200$ & \\
\hline Nunca ou às vezes & $34(49,3)$ & $35(50,7)$ & $69(34,5)$ & \\
\hline Sempre & $51(38,9)$ & $80(61,1)$ & $131(65,5)$ & $0,160 *$ \\
\hline \multicolumn{5}{|l|}{$\begin{array}{l}\text { Coordenação do cuidado e integração } \\
\text { assistencial }\end{array}$} \\
\hline $\begin{array}{l}\text { Existência de casos de CC nos últimos } 12 \\
\text { meses }\end{array}$ & $n=91$ & $n=127$ & $n=218$ & \\
\hline Não & $70(46,7)$ & $80(53,3)$ & $150(68,8)$ & \\
\hline Sim & $21(30,9)$ & $47(69,1)$ & $68(31,2)$ & $0,029 *$ \\
\hline Existência de casos de HSIL & $n=91$ & $n=131$ & $n=222$ & \\
\hline Não & $54(52,4)$ & $49(47,6)$ & $103(46,4)$ & \\
\hline Sim & $37(31,1)$ & $82(68,9)$ & $119(53,6)$ & $0,001^{*}$ \\
\hline
\end{tabular}

${ }^{*}$ Teste Qui-quadrado de Pearson; ${ }^{\top}$ Teste exato de Fisher; ${ }^{\ddagger}$ A variável sexo só mantém diferença estatística significativa após ajuste para idade e estado conjugal e a variável frequência de oferta do exame citopatológico só mantém diferença estatística significativa após ajuste para sexo, idade e estado conjugal; USF Unidade de Saúde da Família; EqSF - Equipe de Saúde da Família; AE - atenção especializada; SMS - Secretaria Municipal de Saúde; CC - câncer cervicouterino; HSIL - lesão intraepitelial de alto grau. Fonte: elaboração própria com base nos dados da pesquisa.

companheiro (69,8\%; $p<0,001)$. A maioria dos profissionais com tempo de atuação $\geq 2$ anos (70,4\%) possuía pós-graduação, ao passo que apenas $29,6 \%$ daqueles com tempo de atuação $<2$ anos afirmaram ter pós-graduação $(p<0,001)$.

Acerca da organização das unidades e acesso ao exame de rastreamento, a realização de exame citopatológico com frequência quinzenal $(69,6 \%)$ e semanal ou mais $(62,0 \%)$ $p=0,033$ e realização de mutirões para ampliar o acesso são mais frequentes entre profissionais com maior tempo de atuação (62,6\%; $p=0,032)$. Da mesma forma, o registro do exame $(57,9 \%$; $p=0,023$ ) e o monitoramento deste para identificar mulheres que possam estar com coleta atrasada $(63,7 \% ; p<0,001)$ foram 
relatadas como práticas mais comuns entre profissionais com maior tempo (Tabela 3).

A respeito da coordenação do cuidado e integração assistencial, a maioria dos profissionais com tempo de atuação $\geq 2$ anos relatou ter havido casos de mulheres com diagnóstico de CC na sua USF $(69,1 \% ; p=0,029)$ e de HSIL $(68,9 \% ; p=0,001)$ (Tabela 3).

Nas análises ajustadas para as variáveis sexo, idade e estado conjugal (dados não mostrados na tabela), as diferenças estatísticas se mantêm para os indicadores de qualidade: função $(p<0,001)$; pós-graduação $(p<0,001)$; realização de mutirões $(p=0,032)$; registro do exame citopatológico $(p=0,009)$; monitoramento do registro para identificar mulheres com coleta atrasada $(p<0,001)$ existência de casos de CC nos últimos 12 meses $(p=0,008)$; e existência de casos de HSIL $(p=0,001)$. $O$ indicador frequência de oferta do exame citopatológico e a variável sociodemográfica sexo apresentam diferenças estatísticas apenas após o ajuste (Tabela 3).

\section{DISCUSSÃO}

A região de saúde apresentou prevalência do tempo de atuação de profissionais na APS no mesmo município $\geq 2$ anos, pouco acima da metade. Ademais, os achados demonstram diferenças estatisticamente significativas para as categorias de tempo e a função de enfermeiro, possuir pós-graduação, além de frequência de oferta, registro e monitoramento do registro do exame do citopatológico.

Apesar de não haver estudos ou protocolos com períodos adequados definidos, considera-se que maior tempo de atuação oportuniza o cumprimento do atributo de longitudinalidade e formação de vínculo na APS ${ }^{15}$. A retenção de profissionais em territórios rurais, não só no Brasil, como em outros países, por mais de 20 meses, ainda é difícil ${ }^{9-11}$, especialmente de médicos de APS. As consequências são piores resultados de saúde para a população, maiores custos para o sistema de saúde ${ }^{10}$ e quebra ou não formação de vínculo entre profissionais e comunidade ${ }^{12}$.

Para condições crônicas, a longitudinalidade do cuidado no âmbito das redes de atenção é fundamental ${ }^{6}$, permitindo uma atuação da EqSF baseada na orientação familiar e comunitária ${ }^{26}$. $\mathrm{Na}$ linha de cuidado estudada, tal fato se torna primordial na prestação do serviço, visto que o exame do Papanicolau é acompanhando de diversos tabus e preceitos culturais ${ }^{18}$, que criam barreiras ao rastreamento ${ }^{17}$. Ademais, o receio de mulheres em realizar o procedimento com enfermeiros do sexo masculino ${ }^{9,17}$ e jovens ${ }^{18}$ pode ser superado, pois a confiança e a adesão ao tratamento aumentam quando o acompanhamento é feito pelo mesmo profissional ao longo do tempo ${ }^{14}$.

Observou-se uma maior prevalência, com diferença estatística significativa, de enfermeiros atuando na atenção primária do mesmo município por maior tempo, comparado aos médicos. A dificuldade de fixação e atração de profissionais ainda é um grande desafio, especialmente em relação aos médicos no Nordeste $^{13}$. O tempo médio de permanência nas unidades de saúde da família é maior entre os enfermeiros ${ }^{20}$.
A ampliação das funções do profissional de enfermagem é debatida em diversos paíse ${ }^{21} \mathrm{e}$, para a linha de cuidado do CC, normalmente é o membro da equipe mais envolvido na busca, rastreamento e acolhimento das demandas das usuárias ${ }^{16}$.

O seguimento do cuidado é bastante provável, quando as mulheres são acompanhadas por enfermeiros ${ }^{19}$, por buscarem ambiente seguro para realizar o exame de rastreamento, bem como sentimentos de valorização e confiança ${ }^{17}$. É necessário, portanto, relação terapêutica baseada na manutenção do vínculo e encorajamento de usuárias a se tornarem protagonistas dos seus cuidados em saúde, resultando em atenção adequada. Enfermeiros treinados adequadamente produzem cuidados de alta qualidade e alcançam bons resultados para a saúde do paciente, sendo tão resolutivos quanto médicos ${ }^{21}$.

A capacitação dos profissionais para ofertar ações apropriadas é outro fator importante na qualidade dos cuidados em saúde ${ }^{21} \mathrm{e}$ necessita de investimentos no âmbito da APS ${ }^{27}$. Nesse sentido, profissionais com maior tempo de atuação tiveram mais chances de possuir pós-graduação. Tal fato evidencia a maior probabilidade de vínculos estáveis se configurarem como estímulo a profissionais se sentirem motivados a se qualificarem. Equipes mais capacitadas desenvolvem os atributos de longitudinalidade ${ }^{28}$ e integralidade, sendo mais bem avaliadas por usuário ${ }^{20}$, exercendo a função de filtro para os serviços especializados, evitando burocratização e encaminhamentos excessivos e desnecessários ${ }^{14}$.

O tempo de atuação também mostrou diferenças para fatores organizacionais de estrutura e processo, como frequência de oferta do citopatológico, realização de mutirões, registro e monitoramento frequente do registro, em que houve maiores prevalências para o tempo de dois anos ou mais. Profissionais que formam vínculo com a unidade de saúde e atuam a mais tempo podem ter processo de trabalho mais adequado ${ }^{7,12}$. A realização de mutirões nas USF é uma estratégia adotada a fim de ampliar o acesso e melhorar o rastreamento, minimizando barreiras ${ }^{18}$. Por outro lado, a ausência de triagem populacional e manutenção de controle das mulheres examinadas através de algum tipo de registro demonstram promover desigualdades em saúde, além de menor eficácia e eficiência do sistema ${ }^{29}$.

A melhor organização da equipe ${ }^{12} \mathrm{e}$ o registro com monitoramento constante ${ }^{29}$ permitem a construção e a manutenção de um elo entre a equipe e as usuárias, revelando a implicação dos profissionais com a comunidade ${ }^{18,28}$. A relação contínua entre a equipe e as mulheres depende de estratégias de monitoramento continuado e aprimoramento tenaz dos componentes de estrutura e processo dos serviços ${ }^{13,30}$, especialmente no atendimento de mulheres mais vulneráveis ao $\mathrm{CC}^{4,17,22}$.

Nota-se a imprescindibilidade de serviços de APS mais organizados, de qualidade e que sejam resolutivos ${ }^{27}$, ao constatar os casos de CC e de HSIL, ainda bastante prevalentes nos territórios ${ }^{1,2,29}$. Houve significância estatística entre tempo $\geq 2$ anos e relatos de HSIL e de CC nos últimos doze meses. Tais achados podem estar relacionados à maior prevalência em mulheres rurais, em decorrência da complexidade dos territórios ou do fato de haver mulheres super-rastreadas ${ }^{18,22}$, enquanto muitas 
obtêm o diagnóstico tardio. Ressalta-se que os profissionais que permanecem no mesmo serviço por mais tempo têm maiores chances de se deparar com algum caso de CC ou HSIL. É necessária a realização de estudos mais aprofundados, para melhor compreensão desses fenômenos.

\section{CONCLUSÕES E IMPLICAÇÕES PARA A PRÁTICA}

O maior tempo de atuação profissional possui maiores prevalências dos indicadores de qualidade das ações para controle do CC. Os achados deste estudo evidenciam a necessidade de um programa integral de rastreamento com qualidade, que tenha como porta de entrada a APS na perspectiva da regionalização, bem como financiamento adequado, para um sistema de saúde universal. Os territórios rurais e remotos apresentam características peculiares, acumulando grande número lesões e de mortes por câncer cervical em mulheres, enfrentando ainda alta rotatividade de profissionais. Tais fatores impactam na qualidade dos serviços e salientam a necessidade de políticas de atração e fixação de profissionais eficazes e adequadas aos contextos dos diversos países.

Como limitação deste estudo, deve-se considerar que o delineamento transversal não fornece inferência causal e o tipo de análise proposto não permite estabelecer relações de associação. Contudo, os indícios sugerem fragilidades contundentes na qualidade dos serviços de APS.

Sugerem-se, fortemente, a gestores e formuladores de políticas, a adoção de medidas para qualificação da mão de obra em saúde, a estruturação de plano de carreira profissional para fornecer mais segurança e estabilidade, podendo impactar em serviços de maior qualidade e redução de mortes evitáveis. Propõe-se uma maior valorização do papel do profissional enfermeiro, que tem grande potencial para otimizar a resolutividade e eficiência dos serviços, especialmente no âmbito da APS. Recomendamse que novos estudos sejam realizados, para elucidar melhor os dados apontados.

\section{AGRADECIMENTOS}

Às coordenadoras da APS dos 19 municípios, que colaboraram na articulação com os profissionais e na logística às Unidades de Saúde. Ao Observatório Baiano de Redes de Atenção à Saúde (OBRAS), que é um grupo de pesquisa do CNPQ (dgp.cnpq. br/dgp/espelhogrupo/9684320673978024), pela contribuição da equipe de coletadores e em grupo de estudos para debate dos resultados.

\section{FINANCIAMENTO}

O presente trabalho foi realizado com apoio da Coordenação de Aperfeiçoamento de Pessoal de Nível Superior - Brasil (CAPES) - Código de Financiamento 001, bolsa de mestrado, concedida a Eduarda Ferreira dos Anjos. Fundação de Amparo à Pesquisa do Estado da Bahia (FAPESB), concedido a Adriano Maia dos
Santos, desenvolvimento do projeto de pesquisa "Estratégias de planejamento e gestão para integração assistencial e garantia de acesso aos serviços especializados de média densidade tecnológica na região de saúde de Vitória da Conquista, Bahia", Edital № 08/2015 - Apoio a Projetos de Pesquisa para Jovem Cientista no Estado da Bahia, Processo no JCB003/2016.

\section{CONTRIBUIÇÕES DOS AUTORES}

Desenho do estudo. Eduarda Ferreira dos Anjos. Kaue Batista Andrade. Poliana Cardoso Martins. Adriano Maia dos Santos.

Coleta de dados. Eduarda Ferreira dos Anjos. Kaue Batista Andrade.

Análise de dados. Eduarda Ferreira dos Anjos. Kaue Batista Andrade. Adriano Maia dos Santos.

Interpretação dos resultados. Eduarda Ferreira dos Anjos. Kaue Batista Andrade. Adriano Maia dos Santos.

Redação e revisão crítica do manuscrito. Eduarda Ferreira dos Anjos. Kaue Batista Andrade. Poliana Cardoso Martins. Jamille Amorim Carvalho Paiva. Nília Maria de Brito Lima Prado. Adriano Maia dos Santos.

Aprovação da versão final do artigo. Eduarda Ferreira dos Anjos. Kaue Batista Andrade. Poliana Cardoso Martins. Jamille Amorim Carvalho Paiva. Nília Maria de Brito Lima Prado. Adriano Maia dos Santos.

Responsabilidade por todos os aspectos do conteúdo e a integridade do artigo publicado.

Eduarda Ferreira dos Anjos. Kaue Batista Andrade. Poliana Cardoso Martins. Jamille Amorim Carvalho Paiva. Nília Maria de Brito Lima Prado. Adriano Maia dos Santos.

\section{EDITOR ASSOCIADO}

Stela Maris de Melo Padoin (1)

\section{EDITOR CIENTÍFICO}

Ivone Evangelista Cabral (1)

\section{REFERÊNCIAS}

1. Fitzmaurice C, Abate D, Abbasi N, Abbastabar H, Abd-Allah F, AbdelRahman $O$ et al. Global, regional, and national cancer incidence, mortality, years of life lost, years lived with disability, and disability-adjusted lifeyears for 29 cancer groups, 1990 to 2017: a systematic analysis for the Global Burden of Disease Study. JAMA Oncol. 2019;5(12):1749-68. http://dx.doi.org/10.1001/jamaoncol.2019.2996. PMid:31560378.

2. Arbyn M, Weiderpass E, Bruni L, de Sanjosé S, Saraiya M, Ferlay $J$ et al. Estimates of incidence and mortality of cervical cancer in 2018 : a worldwide analysis. Lancet Glob Health. 2020;8(2):e191-203. http:// dx.doi.org/10.1016/S2214-109X(19)30482-6. PMid:31812369.

3. Olusola P, Banerjee HN, Philley JV, Dasgupta S. Human Papilloma virusassociated cervical cancer and health disparities. Cells. 2019;8(6):622 http://dx.doi.org/10.3390/cells8060622. PMid:31234354.

4. Karadag Arli S, Bakan AB, Aslan G. Distribution of cervical and breast cancer risk factors in women and their screening behaviours. Eur $J$ Cancer Care. 2019;28(2):e12960. http://dx.doi.org/10.1111/ecc.12960. PMid:30421468. 
5. Instituto Nacional do Câncer, Coordenação de Prevenção e Vigilância, Divisão de Detecção Precoce e Apoio à Organização de Rede. Diretrizes brasileiras para o rastreamento do câncer do colo do útero [Internet]. $2^{\mathrm{a}}$ ed. Rio de Janeiro: INCA;2016. 114 p. [citado 2020 jul 23]. Disponível em: https://www.inca.gov.br/sites/ufu.sti.inca.local/files//media/document// diretrizesparaorastreamentodocancerdocolodoutero_2016_corrigido. pdf

6. Sarfati D, Dyer R, Sam FA, Barton M, Bray F, Buadromo E et al. Cancer control in the Pacific: big challenges facing small island states. Lancet Oncol. 2019;20(9):e475-92. http://dx.doi.org/10.1016/S14702045(19)30400-0. PMid:31395476.

7. Anjos EF, Martins PC, Prado NMBL, Bezerra VM, Almeida PF, Santos AM. Monitoring of cervical cancer control actions and associated factors. Texto Contexto Enferm. 2021;30:e20200254. http://dx.doi. org/10.1590/1980-265x-tce-2020-0254.

8. Stumbar SE, Stevens M, Feld Z. Cervical cancer and its precursors: a preventative approach to screening, diagnosis, and management. Prim Care. 2019;46(1):117-34. http://dx.doi.org/10.1016/j.pop.2018.10.011 PMid:30704652.

9. Ojakaa D, Olango S, Jarvis J. Factors affecting motivation and retention of primary health care workers in three disparate regions in Kenya. Hum Resour Health. 2014;12(1):33. http://dx.doi.org/10.1186/1478-449112-33. PMid:24906964.

10. Russell DJ, McGrail MR, Humphreys JS. Determinants of rural Australian primary health care worker retention: a synthesis of key evidence and implications for policymaking. Aust J Rural Health. 2017;25(1):5-14. http://dx.doi.org/10.1111/ajr.12294. PMid:27087590.

11. Vázquez ML, Vargas I, Garcia-Subirats I, Unger JP, De Paepe P, Mogollón-Pérez AS et al. Doctors' experience of coordination across care levels and associated factors: a cross-sectional study in public healthcare networks of six Latin American countries. Soc Sci Med. 2017;182:10-9. http://dx.doi.org/10.1016/j.socscimed.2017.04.001. PMid:28411523.

12. Muramoto FT, Matumoto S. Repercussions of the Brazilian Program for the Assessment of Quality of Primary Care. Rev Cubana Enferm [Internet]. 2019; [citado 2021 maio 1];35(3):1-17. Disponível em: http:// www.revenfermeria.sld.cu/index.php/enf/article/view/2208

13. Gonçalves RF, Bezerra AFB, Tanaka OY, Santos CRD, Silva KSBE, Sousa IMC. Influence of the Mais Médicos (More Doctors) Program on health services access and use in Northeast Brazil. Rev Saude Publica. 2019;53:110. http://dx.doi.org/10.11606/S1518-8787.2019053001571. PMid:31826176.

14. Lima JG, Giovanella L, Fausto MCR, Bousquat A, Silva EV. Essential attributes of Primary Health Care: national results of PMAQ-AB. Saúde Debate. 2018;42(spe1):52-66. http://dx.doi.org/10.1590/0103$11042018 s 104$

15. Viana MRP, Moura MEB, Nunes BMVT, Monteiro CFS, Lago EC Nursing education for prevention of cervical câncer. Rev Enferm UERJ [Internet]. 2013; [citado 2021 maio 1];21(spe):624-30. Disponível em: https://www.e-publicacoes.uerj.br/index.php/enfermagemuerj/article/ view/10038

16. Perks J, Algoso M, Peters K. Nurse practitioner (NP) led care: cervical screening practices and experiences of women attending a women's health centre. Collegian. 2018;25(5):493-9. http://dx.doi.org/10.1016/j. colegn.2017.12.007.

17. Fernandes NFS, Galvão JR, Assis MMA, Almeida PF, Santos AM. Access to uterine cervical cytology in a health region: invisible women and vulnerable bodies. Cad Saude Publica. 2019;35(10):e00234618. http://dx.doi.org/10.1590/0102-311x00234618. PMid:31596403.
18. World Health Organization. Building the primary health care workforce of the 21st century [Internet]. Geneva:WHO;2018.25 p. (Technical Series on Primary Health Care) [citado 2021 ago 3]. Disponível em: https:/ www.who.int/docs/default-source/primary-health-care-conference/ workforce.pdf

19. Laurant M, van der Biezen M, Wijers N, Watananirun K, Kontopantelis E, van Vught AJ. Nurses as substitutes for doctors in primary care. Cochrane Database Syst Rev. 2018;16(7):CD001271. http://dx.doi. org/10.1002/14651858.CD001271.pub3.

20. Leão CD, Caldeira AP. Assessment of the association between the qualification of physicians and nurses in primary healthcare and the quality of care. Cien Saude Colet. 2011;16(11):4415-23. http://dx.doi. org/10.1590/S1413-81232011001200014. PMid:22124822.

21. Toso BRGO, Filippon J, Giovanella L. Nurses' performance on primary care in the National Health Service in England. Rev Bras Enferm 2016;69(1):182-91. http://dx.doi.org/10.1590/0034-7167.2016690124i. PMid:26871232.

22. Galvão JR, Almeida PF, Santos AM, Bousquat A. Healthcare trajectories and obstacles faced by women in a health region in Northeast Brazil. Cad Saude Publica. 2019;35(12):e00004119. http://dx.doi.org/10.1590/010231100004119. PMid:31800777.

23. Ministério da Saúde (BR), Secretaria de Atenção em Saúde, Departamento de Atenção Básica. Controle dos cânceres do colo do útero e da mama [Internet]. Brasília: Ministério da Saúde; 2013. 124 p. [citado 2020 jul 23]. Disponível em: http://bvsms.saude.gov.br/bvs/ publicacoes/controle_canceres_colo_utero_2013.pdf

24. Bottari CMS, Vasconcellos MM, Mendonça MHM. Cervical cancer as a tracer condition: a proposal for evaluation of primary health care. Cad Saude Publica. 2008;24(Supl. 1):111-22. http://dx.doi.org/10.1590/ S0102-311X2008001300016. PMid:18660896.

25. Souza MKB, Almeida PF, Santos AM, Santos DB, Martins Júnior DF. Estratégias e métodos da pesquisa sobre a Atenção Primária à Saúde na coordenação do cuidado em redes regionalizadas. In: Almeida PF, Santos AM, Souza MKB, organizadores. Atenção primária à saúde na coordenação do cuidado em regiões de saúde. Salvador: Edufba; 2015. p. 117-45. http://dx.doi.org/10.7476/9788523218768.0007.

26. Augusto DK, Lima-Costa MF, Macinko J, Peixoto SV. Factors associated with the evaluation of quality of primary health care by older adults living in the Metropolitan Region of Belo Horizonte, Minas Gerais, Brazil, 2010 Epidemiol Serv Saude. 2019;28(1):e2018128. http://dx.doi.org/10.5123/ S1679-49742019000100017. PMid:30970074.

27. Li X, Krumholz HM, Yip W, Cheng KK, De Maeseneer J, Meng Q et al. Quality of primary health care in China: challenges and recommendations. Lancet. 2020;395(10239):1802-12. http://dx.doi.org/10.1016/S01406736(20)30122-7. PMid:32505251.

28. Nicula FA, Anttila A, Neamtiu L, Zakelj MP, Tachezy R, Chil A et al. Challenges in starting organised screening programmes for cervica cancer in the new member states of the European Union. Eur J Cancer. 2009;45(15):2679-84. http://dx.doi.org/10.1016/j.ejca.2009.07.025 PMid:19699083.

29. Lima EFA, Sousa Al, Leite FMC, Lima RCD, Nascimento MH, Primo $\mathrm{CC}$. Evaluation of the family healthcare strategy from the perspective of health professionals. Esc Anna Nery. 2016;20(2):275-80. http://dx.doi. org/10.5935/1414-8145.20160037.

30. Vidal TB, Tesser CD, Harzheim E, Fontanive PVN. Evaluation of Primary Health Care performance in Florianopolis, Santa Catarina, Brazil, 2012: a cross-sectional population-based study. Epidemio Serv Saude. 2018;27(4):e2017504. http://dx.doi.org/10.5123/s167949742018000400006. PMid:30427399. 